\title{
On Prime Near-Rings with Generalized Derivation
}

\author{
Howard E. Bell \\ Department of Mathematics, Faculty of Mathematics and Science, Brock University, \\ St. Catharines, Ontario, Canada L2S 3A1
}

Correspondence should be addressed to Howard E. Bell, hbell@brocku.ca

Received 29 November 2007; Accepted 25 February 2008

Recommended by Francois Goichot

Let $N$ be a 3-prime 2-torsion-free zero-symmetric left near-ring with multiplicative center $Z$. We prove that if $N$ admits a nonzero generalized derivation $f$ such that $f(N) \subseteq Z$, then $N$ is a commutative ring. We also discuss some related properties.

Copyright (C) 2008 Howard E. Bell. This is an open access article distributed under the Creative Commons Attribution License, which permits unrestricted use, distribution, and reproduction in any medium, provided the original work is properly cited.

\section{Introduction}

Let $N$ be a zero-symmetric left near-ring, not necessarily with a multiplicative identity element; and let $Z$ be its multiplicative center. Define $N$ to be 3-prime if for all $a, b \in N \backslash\{0\}, a N b \neq\{0\}$; and call $N$ 2-torsion-free if $(N,+)$ has no elements of order 2 . A derivation on $N$ is an additive endomorphism $D$ of $N$ such that $D(x y)=x D(y)+D(x) y$ for all $x, y \in N$. A generalized derivation $f$ with associated derivation $D$ is an additive endomorphism $f: N \rightarrow N$ such that $f(x y)=f(x) y+x D(y)$ for all $x, y \in N$. In the case of rings, generalized derivations have received significant attention in recent years.

In [1], we proved the following.

Theorem A. If $N$ is 3-prime and 2-torsion-free and $D$ is a derivation such that $D^{2}=0$, then $D=0$.

Theorem B. If $N$ is a 3-prime 2-torsion-free near-ring which admits a nonzero derivation $D$ for which $D(N) \subseteq Z$, then $N$ is a commutative ring.

Theorem C. If $N$ is a 3-prime 2-torsion-free near-ring admitting a nonzero derivation $D$ such that $D(x) D(y)=D(y) D(x)$ for all $x, y \in N$, then $N$ is a commutative ring.

In this paper, we investigate possible analogs of these results, where $D$ is replaced by a generalized derivation $f$. 
We will need three easy lemmas.

Lemma 1.1 (see [1, Lemma 3]). Let $N$ be a 3-prime near-ring.

(i) If $z \in Z \backslash\{0\}$, then $z$ is not a zero divisor.

(ii) If $Z \backslash\{0\}$ contains an element $z$ such that $z+z \in Z$, then $(N,+)$ is abelian.

(iii) If $D$ is a nonzero derivation and $x \in N$ is such that $x D(N)=\{0\}$ or $D(N) x=\{0\}$, then $x=0$.

Lemma 1.2 (see [2, Proposition 1]). If $N$ is an arbitrary near-ring and $D$ is a derivation on $N$, then $D(x y)=D(x) y+x D(y)$ for all $x, y \in N$.

Lemma 1.3. Let $N$ be an arbitrary near-ring and let $f$ be a generalized derivation on $N$ with associated derivation $D$. Then

$$
(f(a) b+a D(b)) c=f(a) b c+a D(b) c \quad \forall a, b, c \in N .
$$

Proof. Clearly $f((a b) c)=f(a b) c+a b D(c)=(f(a) b+a D(b)) c+a b D(c)$; and by using Lemma 1.2, we obtain $f(a(b c))=f(a) b c+a D(b c)=f(a) b c+a D(b) c+a b D(c)$.

Comparing these two expressions for $f(a b c)$ gives the desired conclusion.

\section{The main theorem}

Our best result is an extension of Theorem B.

Theorem 2.1. Let $N$ be a 3-prime 2-torsion-free near-ring. If $N$ admits a nonzero generalized derivation $f$ such that $f(N) \subseteq Z$, then $N$ is a commutative ring.

In the proof of this theorem, as well as in a later proof, we make use of a further lemma.

Lemma 2.2. Let $R$ be a 3-prime near-ring, and let $f$ be a generalized derivation with associated derivation $D \neq 0$. If $D(f(N))=\{0\}$, then $f(D(N))=\{0\}$.

Proof. We are assuming that $D(f(x))=0$ for all $x \in N$. It follows that $D(f(x y))=D(f(x) y)+$ $D(x D(y))=0$ for all $x, y \in N$, that is,

$$
f(x) D(y)+D(x) D(y)+x D^{2}(y)=0 \quad \forall x, y \in N .
$$

Applying $D$ again, we get

$$
f(x) D^{2}(y)+D^{2}(x) D(y)+D(x) D^{2}(y)+D(x) D^{2}(y)+x D^{3}(y)=0 \quad \forall x, y \in N .
$$

Taking $D(y)$ instead of $y$ in (2.1) gives $f(x) D^{2}(y)+D(x) D^{2}(y)+x D^{3}(y)=0$, hence (2.2) yields

$$
D^{2}(x) D(y)+D(x) D^{2}(y)=0 \quad \forall x, y \in N .
$$

Now, substitute $D(x)$ for $x$ in (2.1), obtaining $f(D(x)) D(y)+D^{2}(x) D(y)+D(x) D^{2}(y)=0$; and use (2.3) to conclude that $f(D(x)) D(y)=0$ for all $x, y \in N$. Thus, by Lemma 1.1(iii), $f(D(x))=0$ for all $x \in N$. 
Proof of Theorem 2.1. Since $f \neq 0$, there exists $x \in N$ such that $0 \neq f(x) \in Z$. Since $f(x)+f(x)=$ $f(x+x) \in Z,(N,+)$ is abelian by Lemma 1.1(ii). To complete the proof, we show that $N$ is multiplicatively commutative.

First, consider the case $D=0$, so that $f(x y)=f(x) y \in Z$ for all $x, y \in N$. Then $f(x) y w=$ $w f(x) y$, hence $f(x)(y w-w y)=0$ for all $x, y, w \in N$. Choosing $x$ such that $f(x) \neq 0$ and invoking Lemma 1.1(i), we get $y w-w y=0$ for all $y, w \in N$.

Now assume that $D \neq 0$, and let $c \in Z \backslash\{0\}$. Then $f(x c)=f(x) c+x D(c) \in Z$; therefore, $(f(x) c+x D(c)) y=y(f(x) c+x D(c))$ for all $x, y \in N$, and by Lemma 1.3, we see that $f(x) c y+$ $x D(c) y=y f(x) c+y x D(c)$. Since both $f(x)$ and $D(c)$ are in $Z$, we have $D(c)(x y-y x)=0$ for all $x, y \in N$, and provided that $D(Z) \neq\{0\}$, we can conclude that $N$ is commutative.

Assume now that $D \neq 0$ and $D(Z)=\{0\}$. In particular, $D(f(x))=0$ for all $x \in N$. Note that for $c \in N$ such that $f(c)=0, f(c x)=c D(x) \in Z$; hence by Lemma 2.2, $D(x) D(y) \in Z$ and $D(y) D(x) \in Z$ for each $x, y \in N$. If one of these is 0 , the other is a central element squaring to 0 , hence is also 0 . The remaining possibility is that $D(x) D(y)$ and $D(y) D(x)$ are nonzero central elements, in which case $D(x)$ is not a zero divisor. Thus $D(x) D(x) D(y)=D(x) D(y) D(x)$ yields $D(x)(D(x) D(y)-D(y) D(x))=0=D(x) D(y)-D(y) D(x)$. Consequently, $N$ is commutative by Theorem $C$.

\section{On Theorems $A$ and $C$}

Theorem $\mathrm{C}$ does not extend to generalized derivations, even if $N$ is a ring. As in [3], consider the ring $H$ of real quaternions, and define $f: H \rightarrow H$ by $f(x)=i x+x i$. It is easy to check that $f$ is a generalized derivation with associated derivation given by $D(x)=x i-i x$, and that $f(x) f(y)=f(y) f(x)$ for all $x, y \in H$.

Theorem A also does not extend to generalized derivations, as we see by letting $N$ be the ring $M_{2}(F)$ of $2 \times 2$ matrices over a field $F$ and letting $f$ be defined by $f(x)=e_{12} x$. However, we do have the following results.

Theorem 3.1. Let $N$ be a 3-prime near-ring, and let $f$ be a generalized derivation on $N$ with associated derivation $D$. If $f^{2}=0$, then $D^{3}=0$. Moreover, if $N$ is 2-torsion-free, then $D(Z)=\{0\}$.

Proof. We have

$$
f^{2}(x y)=f(f(x) y+x D(y))=f(x) D(y)+f(x) D(y)+x D^{2}(y)=0 \quad \forall x, y \in N .
$$

Applying $f$ to (3.1) gives

$$
f(x) D^{2}(y)+f(x) D^{2}(y)+f(x) D^{2}(y)+x D^{3}(y)=0 \quad \forall x, y \in N .
$$

Substituting $D(y)$ for $y$ in (3.1) gives

$$
f(x) D^{2}(y)+f(x) D^{2}(y)+x D^{3}(y)=0 ;
$$

Therefore, by (3.2) and (3.3),

$$
f(x) D^{2}(y)=0 \quad \forall x, y \in N .
$$

It now follows from (3.3) that $x D^{3}(y)=0$ for all $x, y \in N$; and since $N$ is 3-prime, $D^{3}=0$. 
Suppose now that $N$ is 2-torsion-free and that $D(Z) \neq\{0\}$, and let $z \in Z$ be such that $D(z) \neq 0$. Then if $x, y \in N$ and $f(N) x=\{0\}$, then $f(y z) x=f(y) z x+y D(z) x=0=y D(z) x$; and since $N$ is 3-prime and $D(z)$ is not a zero divisor, $x=0$. It now follows from (3.4) that $D^{2}=0$ and hence by Theorem A that $D=0$. But this contradicts our assumption that $D(Z) \neq\{0\}$, hence $D(Z)=\{0\}$ as claimed.

Theorem 3.2. Let $N$ be a 3-prime and 2-torsion-free near-ring with 1. Iff is a generalized derivation on $N$ such that $f^{2}=0$ and $f(1) \in Z$, then $f=0$.

Proof. Note that $f(x)=f(1 x)=f(1) x+1 D(x)$, so

$$
f(x)=c x+D(x), \quad c \in Z
$$

If $c=0$, then $f=D$ and $D^{2}=0$, so $D=0$ by Theorem A and therefore $f=0$.

If $c \neq 0$, then $c$ is not a zero divisor, hence by (3.4) $D^{2}=0$ and $D=0$. But then $f(x)=c x$ and $f^{2}(x)=c^{2} x=0$ for all $x \in N$. Since $c^{2}$ is not a zero divisor, we get $N=\{0\}-\mathrm{a}$ contradiction. Thus, $c=0$ and we are finished.

\section{More on Theorem C}

In [4], the author studied generalized derivations $f$ with associated derivation $D$ which have the additional property that

$$
f(x y)=D(x) y+x f(y) \quad \forall x, y \in N
$$

Our final theorem, a weak generalization of Theorem C, was stated in [4]; but the proof given was not correct. (At one point, both left and right distributivity were assumed.) We now have all the results required for a proof.

Theorem 4.1. Let $N$ be a 3-prime 2-torsion-free near-ring which admits a generalized derivation $f$ with nonzero associated derivation $D$ such that $f$ satisfies $(*)$. If $f(x) f(y)=f(y) f(x)$ for all $x, y \in N$, then $N$ is a commutative ring.

Proof. It is correctly shown in [4] that $(N,+)$ is abelian and either $f(N) \subseteq Z$ or $D(f(N))=\{0\}$. Hence, in view of Theorem 2.1, we may assume that $D(f(N))=0$ and therefore, by Lemma 2.2, that $f(D(N))=\{0\}$. We calculate $f(D(x) D(y))$ in two ways. Using the defining property of $f$, we obtain $f(D(x) D(y))=f(D(x)) D(y)+D(x) D^{2}(y)=D(x) D^{2}(y)$; and using $(*)$, we obtain $f(D(x) D(y))=D^{2}(x) D(y)+D(x) f(D(y))=D^{2}(x) D(y)$. Thus, $D^{2}(x) D(y)=D(x) D^{2}(y)$ for all $x, y \in N$. But since $D(f(N))=\{0\},(2.3)$ holds in this case as well; therefore $D^{2}(x) D(y)=0$ for all $x, y \in N$, hence by Lemma 1.1(iii) $D^{2}=0$. Thus, $D=0$, contrary to our original hypothesis, so that the case $D(f(N))=\{0\}$ does not in fact occur.

\section{Acknowledgment}

This research is supported by the Natural Sciences and Engineering Research Council of Canada, Grant no. 3961. 


\section{References}

[1] H. E. Bell and G. Mason, "On derivations in near-rings," in Near-Rings and Near-Fields (Tübingen, 1985), G. Betsch, Ed., vol. 137 of North-Holland Mathematics Studies, pp. 31-35, North-Holland, Amsterdam, The Netherlands, 1987.

[2] X.-K. Wang, "Derivations in prime near-rings," Proceedings of the American Mathematical Society, vol. 121, no. 2, pp. 361-366, 1994.

[3] H. E. Bell and N.-U. Rehman, "Generalized derivations with commutativity and anti-commutativity conditions," Mathematical Journal of Okayama University, vol. 49, no. 1, pp. 139-147, 2007.

[4] Ö. Gölbaşi, "Notes on prime near-rings with generalized derivation," Southeast Asian Bulletin of Mathematics, vol. 30, no. 1, pp. 49-54, 2006. 


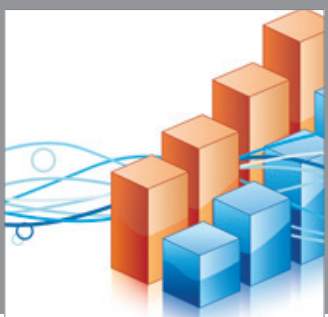

Advances in

Operations Research

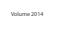

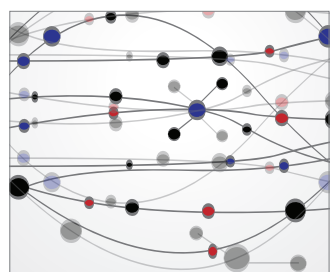

\section{The Scientific} World Journal
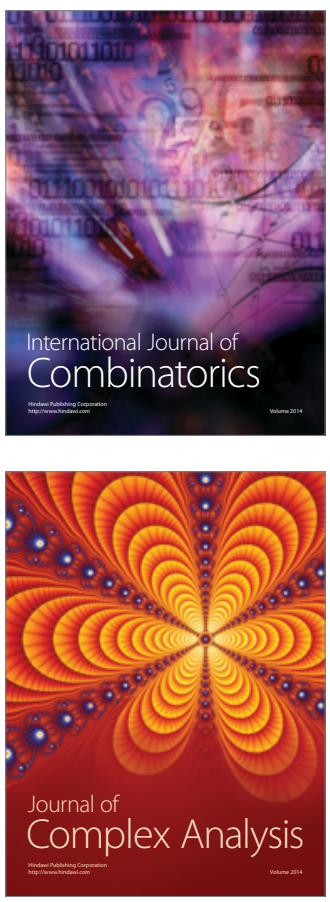

International Journal of

Mathematics and

Mathematical

Sciences
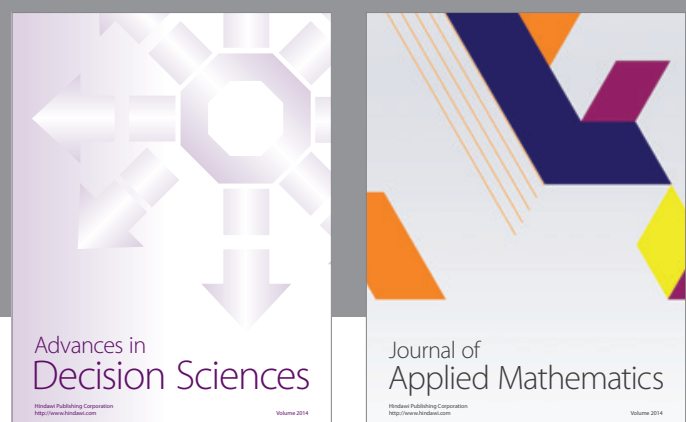

Journal of

Applied Mathematics
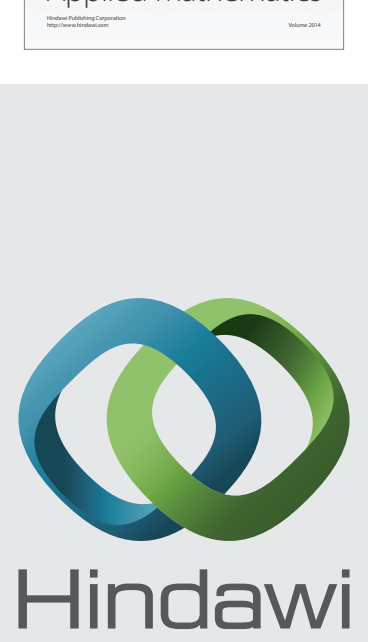

Submit your manuscripts at http://www.hindawi.com
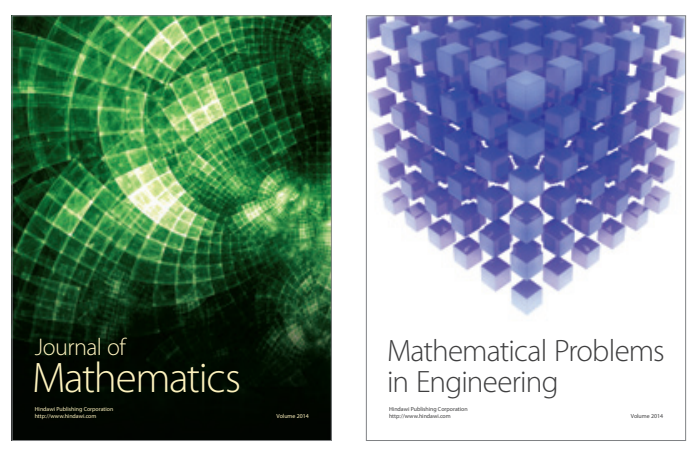

Mathematical Problems in Engineering
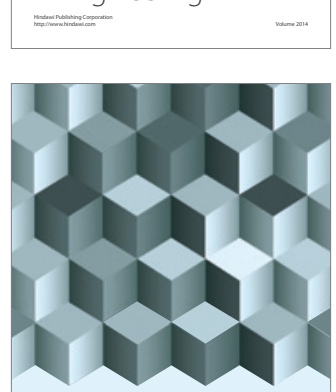

Journal of

Function Spaces
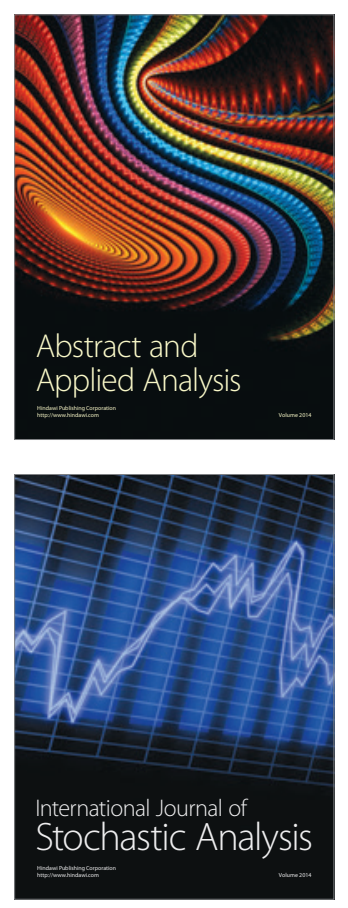

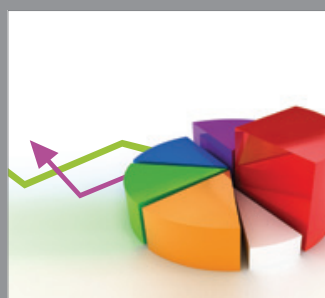

ournal of

Probability and Statistics

Promensencen
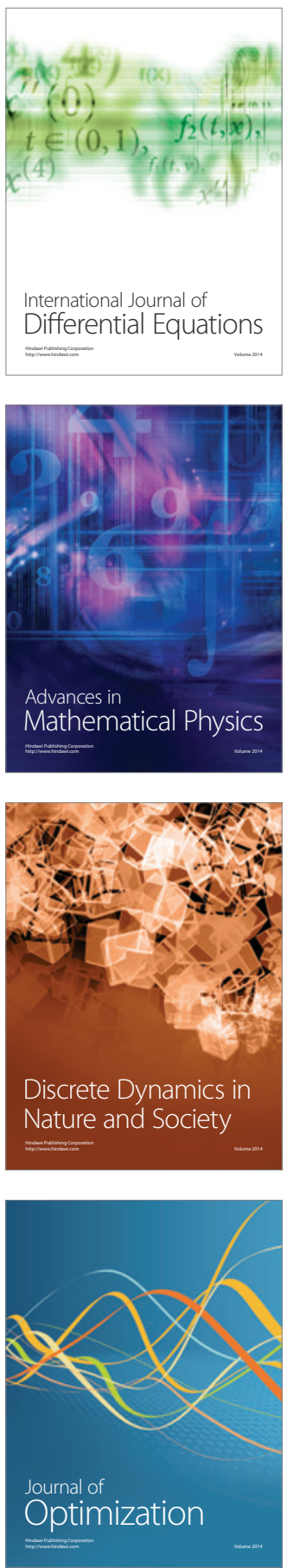